\title{
PERCOLATION ON THE INFORMATION- THEORETICALLY SECURE SIGNAL TO INTERFERENCE RATIO GRAPH
}

\author{
RAHUL VAZE, * Tata Institute of Fundamental Research \\ SRIKANTH IYER, ${ }^{* *}$ Indian Institute of Science
}

\begin{abstract}
We consider a continuum percolation model consisting of two types of nodes, namely legitimate and eavesdropper nodes, distributed according to independent Poisson point processes in $\mathbb{R}^{2}$ of intensities $\lambda$ and $\lambda_{E}$, respectively. A directed edge from one legitimate node $A$ to another legitimate node $B$ exists provided that the strength of the signal transmitted from node $A$ that is received at node $B$ is higher than that received at any eavesdropper node. The strength of the signal received at a node from a legitimate node depends not only on the distance between these nodes, but also on the location of the other legitimate nodes and an interference suppression parameter $\gamma$. The graph is said to percolate when there exists an infinitely connected component. We show that for any finite intensity $\lambda_{E}$ of eavesdropper nodes, there exists a critical intensity $\lambda_{c}<\infty$ such that for all $\lambda>\lambda_{c}$ the graph percolates for sufficiently small values of the interference parameter. Furthermore, for the subcritical regime, we show that there exists a $\lambda_{0}$ such that for all $\lambda<\lambda_{0} \leq \lambda_{c}$ a suitable graph defined over eavesdropper node connections percolates that precludes percolation in the graphs formed by the legitimate nodes.
\end{abstract}

Keywords: Percolation; information-theoretic security; wireless communication; SINR graph

2010 Mathematics Subject Classification: Primary 60D05; 60G70

Secondary $05 \mathrm{C} 05 ; 90 \mathrm{C} 27$

\section{Introduction and main results}

Random geometric graphs have been used extensively to study various properties of wireless communication networks. The nodes of the graph represent the communicating entities that are assumed to be randomly distributed in space, and the edges/connections between nodes reflect the realistic wireless communication links. With the simplest connection model, two nodes are connected (or have an edge between them) provided they are within a specified cutoff distance from each other [6], [7]. Another connection model of interest is the protocol model [4], that incorporates interference emanating from simultaneous transmission by multiple nodes, where two nodes are connected if there is no other node in a specified cutoff area (guard zone) around the two nodes. Thus, a smaller cutoff area results in greater spatial reuse, or more nodes being able to communicate simultaneously. A non-guard-zone-based connection model for wireless communication is the threshold model [4], where two nodes are connected if the signal-to-noise ratio (SINR) between them is more than a certain threshold. The SINR measures the strength of

Received 25 January 2013; revision received 8 August 2013.

* Postal address: School of Technology and Computer Science, Tata Institute of Fundamental Research, Homi Bhabha Road, Mumbai, 400005, India.

** Postal address: Department of Mathematics, Indian Institute of Science, Bangalore, 560012, India.

Email address: skiyer@math.iisc.ernet.in 
the signal received from a particular node relative to those received from other nodes. The SINR between nodes $X_{i}$ and $X_{j}$ is defined as

$$
\operatorname{SINR}_{i j}=\frac{P \ell\left(X_{i}, X_{j}\right)}{N+\gamma \sum_{k \neq i, j} P \ell\left(X_{k}, X_{j}\right)},
$$

where $P$ is the transmitted power from each node, $\ell\left(X_{i}, X_{j}\right) \leq 1$ is the path-loss or attenuation factor, $N>0$ is the environment noise, and $\gamma \geq 0$ is the interference suppression parameter.

The existence of a path between two nodes in the graph implies the ability of those nodes to communicate via a multi-hop path. Consequently, percolation in the graph corresponds to long-range connectivity among large numbers of nodes that are part of the giant component. Assuming that the nodes are distributed according to a Poisson point process in $\mathbb{R}^{2}$ of intensity $\lambda$, the existence of percolation in the graph with the SINR threshold connection model was shown in [1] and [2] for all sufficiently small $\gamma>0$.

Of recent interest is the problem of percolation in wireless networks in the presence of eavesdropper nodes [5], [8], and [9]. In these models, referred to as the information-theoretic secure models, a legitimate node $i$ is connected (has an edge) to node $j$ provided node $j$ is closer to node $i$ than its nearest eavesdropper. These are the links over which secure communication can take place in the presence of eavesdroppers of arbitrary capability. The existence of phase transition of percolation in these graphs was established in [5], [8], and [9], assuming that the legitimate and eavesdropper nodes are distributed according to independent Poisson point processes in $\mathbb{R}^{2}$ of intensities $\lambda$ and $\lambda_{E}$, respectively. Using a branching process argument, [5] and [9] showed that if the ratio $\lambda / \lambda_{E}<1$ then, almost surely, no unbounded connected component exits.

The above secrecy graph model [5], [8], and [9] assumes that the signals transmitted from different legitimate nodes do not interfere with each other. In reality, that is difficult to incorporate, since there are a large number of legitimate nodes, and all cannot transmit on orthogonal frequency or time slots. To generalize the secrecy graph model, we extend the notion of the secrecy graph using the SINR or threshold model, where two legitimate nodes are connected if the SINR between them is more than the SINR at any other eavesdropper node. We derive two results on the percolation properties for this new SINR-based secrecy graph model. The first result is similar in spirit to the one derived by [2]. It states that for any given intensity $\lambda_{E}$ of the eavesdropper nodes, the secrecy graph percolates for sufficiently large intensity $\lambda$ of legitimate nodes and all sufficiently small interference suppression parameter $\gamma$. The second result is that for a given $\lambda_{E}$ and $\gamma>0$, if the density of legitimate nodes is below a threshold, then the graph does not percolate. To prove the second result, we use a novel technique of defining a suitable graph over eavesdropper node connections, where percolation in the eavesdropper nodes' graph precludes percolation in the graphs formed by the legitimate nodes. To complete the result we show that for any given $\lambda_{E}$ and $\gamma>0$, if the density of legitimate nodes is below a threshold then the defined eavesdropper nodes' graph percolates.

Before we proceed to describe the model in detail and state the main results, we need some notation.

Notation: The cardinality of set $S$ is denoted by $|S|$. The complement of set $S$ is denoted by $\bar{S}$. A ball of radius $r$, centered at $x$, is denoted by $B(x, r)$. The boundary of a set $G \subset \mathbb{R}^{2}$ is denoted by $\delta G$. For a set $A \subset \mathbb{R}^{2}, a+A$ denotes a translation of $A$ with $a \in \mathbb{R}^{2}$ as the center. The Lebesgue measure of a set $A \subset \mathbb{R}^{2}$ is denoted as $v(A)$. 


\subsection{System model}

We now describe the secure SINR graph (SSG), which generalizes the secrecy graph considered in [5], [8], and [9], by allowing all legitimate nodes to transmit at the same time/frequency and interfere with each other's communication. Let $\Phi$ be the set of legitimate nodes, and $\Phi_{E}$ be the set of eavesdropper nodes. We assume that the points in $\Phi$ and $\Phi_{E}$ are distributed according to independent Poisson point processes (PPP) with intensities $\lambda$ and $\lambda_{E}$, respectively. Let $x_{i}, x_{j} \in \Phi$, and $e \in \Phi_{E}$. Without loss of generality, we assume an average power constraint of unity $(P=1)$ at each node in $\Phi$, and noise variance $N=1$. Let $0<\gamma \leq 1$ be the processing gain of the system (interference suppression parameter), which depends on the orthogonality between codes used by different legitimate nodes during simultaneous transmissions. Then the SINR between $x_{i}$ and $x_{j}$ is

$$
\operatorname{SINR}_{i j}:=\frac{\ell\left(x_{i}, x_{j}\right)}{\gamma \sum_{k \in \Phi k \neq i} \ell\left(x_{k}, x_{j}\right)+1},
$$

and between $x_{i}$ and $e$ is

$$
\operatorname{SINR}_{i e}:=\frac{\ell\left(x_{i}, e\right)}{\sum_{k \in \Phi k \neq i} \ell\left(x_{k}, x_{j}\right)+1} .
$$

Note that the parameter $\gamma$ is absent in the second SINR formula. This is due to the fact that the code used by the legitimate nodes is not known to the eavesdroppers, hence, no processing gain can be obtained at any of the eavesdroppers. From [11], the maximum rate of reliable communication between $x_{i}$ and $x_{j}$ such that an eavesdropper $e$ gets no knowledge is given by

$$
R_{i j}^{\mathrm{SINR}}(e):=\left[\log _{2}\left(1+\operatorname{SINR}_{i j}\right)-\log _{2}\left(1+\operatorname{SINR}_{i e}\right)\right]^{+},
$$

and the maximum rate of communication between $x_{i}$ and $x_{j}$ that is secured from all the eavesdropper nodes of $\Phi_{E}$ is

$$
R_{i j}^{\mathrm{SINR}}:=\min _{e \in \Phi_{E}} R_{i j}(e)
$$

Definition 1.1. SINR secrecy graph (SSG) is a directed graph $\operatorname{SSG}(\theta):=\{\Phi, \mathcal{E}\}$, with vertex set $\Phi$ and edge set $\mathcal{E}:=\left\{\left(x_{i}, x_{j}\right): R_{i j}^{\mathrm{SINR}}>\theta\right\}$, where $\theta$ is the minimum rate of secure communication required between any two nodes of $\Phi$.

We will assume $\theta=0$ for the rest of the paper, and represent $\operatorname{SSG}(0)$ as SSG. The results can be generalized easily for $\theta>0$. with $\theta=0$, SSG $:=\{\Phi, \mathcal{E}\}$, and edge set $\mathcal{E}:=\left\{\left(x_{i}, x_{j}\right): \operatorname{SINR}_{i j}>\operatorname{SINR}_{i e}\right.$, for all $\left.e \in \Phi_{E}\right\}$.

Definition 1.2. We define that a node $x_{i}$ can connect to $x_{j}$ (or there is a link/connection between them) if $\left(x_{i}, x_{j}\right) \in \mathrm{SSG}$.

Definition 1.3. We define that there is a path from node $x_{i} \in \Phi$ to $x_{j} \in \Phi$ if there is a connected path from $x_{i}$ to $x_{j}$ in the SSG. A path between $x_{i}$ and $x_{j}$ on SSG is represented as $x_{i} \rightarrow x_{j}$.

Definition 1.4. The connected component of any node $x_{j} \in \Phi$, is defined as $C_{x_{j}}:=\left\{x_{k} \in \Phi\right.$, $\left.x_{j} \rightarrow x_{k}\right\}$, with cardinality $\left|C_{x_{j}}\right|$.

Remark 1.1. Note that because of stationarity of the PPP, the distribution of $\left|C_{x_{j}}\right|$ does not depend on $j$, and, hence, without loss of generality from here on we consider node $x_{1}$ for the purposes of defining connected components. Furthermore, we assume without loss of generality that $x_{1}$ is at the origin.

In this paper we are interested in studying the percolation properties of the SSG. In particular, we are interested in finding the minimum value $\lambda_{c}$ of $\lambda$ for which the probability of having an 
unbounded connected component in SSG is greater than zero, as a function of $\lambda_{E}$, i.e. $\lambda_{c}:=$ $\inf \left\{\lambda: P\left(\left|C_{x_{1}}\right|=\infty\right)>0\right\}$. The event $\left\{\left|C_{x_{1}}\right|=\infty\right\}$ is also referred to as percolation on SSG, and we say that percolation happens if $P\left(\left\{\left|C_{x_{1}}\right|=\infty\right\}\right)>0$, and does not happen if $P\left(\left\{\left|\mathcal{C}_{x_{1}}\right|=\right.\right.$ $\infty\})=0$.

Remark 1.2. Assuming that all legitimate nodes can transmit in orthogonal time/frequency slots then we can use the secrecy graph SG, introduced in [5]. This is where two legitimate nodes are connected if the received signal power between them is more than the received signal power at the nearest eavesdropper, i.e. $S G:=\{\Phi, \mathcal{E}\}$, with vertex set $\Phi$ and edge set $\mathcal{E}:=\left\{\left(x_{i}, x_{j}\right): \ell\left(x_{i}, x_{j}\right)>\ell\left(x_{i}, e\right)\right.$ for all $\left.e \in \Phi_{E}\right\}$. Percolation properties of the SG were studied in [8] and [9], where in [9] it was shown that if $\lambda<\lambda_{E}$ then there is no percolation, while [8] showed the existence of $\lambda$ for any fixed $\lambda_{E}$ for which the SG percolates. The graph structure of the SSG is more complicated compared to the SG because of the presence of interference power terms corresponding to simultaneously transmitting legitimate nodes, and, hence, the results of [8] and [9] do not apply for the SSG. For example, consider the case of $\gamma=0$, where it is possible that two legitimate nodes $x_{i}$ and $x_{j}$, with $d_{i j}>\min _{e \in \Phi_{E}} d_{i e}$ can connect to each other in the SSG, however, $x_{i}$ and $x_{j}$ cannot connect to each other in the SG since $d_{i j}>\min _{e \in \Phi_{E}} d_{i e}$. Similarly, if $x_{j}$ is closer to $x_{i}$ than any other eavesdropper node then $x_{i}$ is connected to $x_{j}$ in the SG, however, that may not be the case in the SSG.

Remark 1.3. Without the presence of eavesdropper nodes, percolation on the SINR graph, where the vertex set is $\Phi$, and edge set $\mathcal{E}:=\left\{\left(x_{i}, x_{j}\right): \mathrm{SINR}_{i j} \geq \beta, x_{i}, x_{j} \in \Phi\right\}$ for some fixed threshold $\beta$, has been studied in [1], [2], and [10]. The results of [1], [2], and [10], however, do not apply for the SSG, since for the $\mathrm{SSG} \beta=\mathrm{SINR}_{i e}$ is a random variable that depends on both $\Phi$ and $\Phi_{E}$.

Remark 1.4. Note that we have defined SSG to be a directed graph, and the connected component of $x_{1}$ is its out-component, i.e. the set of nodes with which $x_{1}$ can communicate secretly. Since $x_{i} \rightarrow x_{j}, x_{i}, x_{j} \in \Phi$, does not imply $x_{j} \rightarrow x_{i} x_{i}, x_{j} \in \Phi$, one can similarly define the in-component $C_{x_{j}}^{i n}:=\left\{x_{k} \in \Phi, x_{k} \rightarrow x_{j}\right\}$, the bi-directional component $C_{x_{j}}^{b d}:=$ $\left\{x_{k} \in \Phi, x_{j} \rightarrow x_{k}\right.$ and $\left.x_{k} \rightarrow x_{j}\right\}$, and either the one-directional component $C_{x_{j}}^{e d}:=\left\{x_{k} \in\right.$ $\Phi, x_{j} \rightarrow x_{k}$ or $\left.x_{k} \rightarrow x_{j}\right\}$. Percolation on $C_{x_{j}}^{i n}, C_{x_{j}}^{b d}$, and $C_{x_{j}}^{e d}$ is in principle similar to the percolation on the out-component, but are not considered in this paper.

\subsection{Main results}

Theorem 1.1. For the signal attenuation function $\ell(x)$, such that $\int x \ell(x) d x<\infty$, for any $\lambda_{E}$, there exists $\lambda^{\prime}<\infty$ and a function $\gamma^{\prime}\left(\lambda, \lambda_{E}\right)>0$, such that $P\left(\left|C_{x_{1}}\right|=\infty\right)>0$ in the SSG for $\lambda>\lambda^{\prime}$ and $\gamma<\gamma^{\prime}\left(\lambda, \lambda_{E}\right)$.

We show that for small enough $\gamma$, there exists a large enough $\lambda$ for which the SSG percolates with positive probability for any value of $\lambda_{E}$. This result is similar in spirit to [1] and [2], where percolation is shown to occur in the SINR graph, where two nodes are connected if the SINR between them is more than a fixed threshold $\beta$ (without the secrecy constraint due to eavesdroppers) for small enough $\gamma$ with finite and unbounded support signal attenuation function, respectively. The major difference between the SSG and SINR graphs, is that with the SSG the threshold for connection between two nodes (maximum of SINRs received at all eavesdroppers) is a random variable that depends on both the legitimate and eavesdropper density; in contrast the threshold in the SINR graph is a fixed constant. 
To prove the result, we consider percolation on another graph, $\mathrm{SSG}^{e}$, that is a subset of the SSG. We obtain $\mathrm{SSG}^{e}$ from SSG by replacing the SINR at each eavesdropper node in the SSG definition by $\operatorname{SINR}_{i e}=\ell\left(d_{i e}\right)$, i.e. the SINR at each eavesdropper node is replaced by just the signal power received at the eavesdropper node and setting the interference power terms equal to zero. Considering this subset $\mathrm{SSG}^{e}$ simplifies the percolation analysis significantly. Then to show the percolation on the subset $\mathrm{SSG}^{e}$, we map the continuum percolation of SSG to an appropriate bond percolation on the square lattice, in a similar approach to [2].

For the converse, we have the following theorem on the lower bound for the critical density $\lambda_{c}$.

Theorem 1.2. For every $\lambda_{E}>0$ and $\gamma \in(0,1)$, there exists $a \lambda_{c}=\lambda_{c}\left(\lambda_{E}, \gamma\right)>0$ such that for all $\lambda<\lambda_{c}, P\left(\left|C_{x_{1}}\right|=\infty\right)=0$ in the SSG.

We show that for any $\gamma>0$, there exists small enough $\lambda$ for which the SSG does not percolate for any value of $\lambda_{E}$. In earlier work on the secrecy graph with no interference among simultaneously transmitting legitimate nodes, a stronger result was proved that if $\lambda<\lambda_{E}$ then the secrecy graph does not percolate [9] using a branching process argument on the out-degree distribution. We are only able to show an existential result for the SSG, since finding the outdegree distribution of any node in the SSG is quite challenging and SSG is not amenable to analysis similar in approach to [9].

The proof idea is to define an appropriate eavesdropper node graph such that if an edge exists between two eavesdropper nodes then there exists no edge of the SSG that crosses that edge in $\mathbb{R}^{2}$. Note that for the SSG to percolate, there should be left-to-right crossing and top-tobottom crossing of any square box of large size in $\mathbb{R}^{2}$ by connected edges of SSG. However, if the eavesdropper node graph percolates then there cannot be left-to-right crossing and topto-bottom crossing of any square box of large size in $\mathbb{R}^{2}$ by connected edges of the SSG, and, consequently, the SSG cannot percolate if the eavesdropper node graph percolates. Then we derive conditions for percolation on the defined eavesdropper node graph to find conditions when the SSG does not percolate.

\section{Proof of Theorem 1.1}

In this section, we are interested in the supercritical regime and want to find an upper bound on $\lambda$ such that $P\left(\left|C_{x_{1}}\right|=\infty\right)>0$ for a fixed $\lambda_{E}$. Towards that end, we will tie up the percolation on the SSG to a bond percolation on a square lattice, and show that bond percolation on the square lattice implies percolation in the SSG.

For the supercritical regime, we consider the enhanced graph $\mathrm{SSG}^{e}$, where $\mathrm{SSG}^{e}:=\left\{\Phi, \mathcal{E}^{e}\right\}$, with edge set $\mathcal{E}^{e}:=\left\{\left(x_{i}, x_{j}\right): \operatorname{SINR}_{i j}>\ell\left(d_{i e}\right)\right.$ for all $\left.e \in \Phi_{E}\right\}$. For defining $\mathrm{SSG}^{e}$, we have considered the interference power at the eavesdropper nodes to be zero. Clearly, $\mathrm{SSG}^{e} \subseteq \mathrm{SSG}$, and, hence, if $\mathrm{SSG}^{e}$ percolates, then so does SSG.

We tile $\mathbb{R}^{2}$ into a square lattice $\boldsymbol{S}$ with side $s$. Let $\boldsymbol{S}^{\prime}=\boldsymbol{S}+(s / 2, s / 2)$ be the dual lattice of $\boldsymbol{S}$ obtained by translating each edge of $\boldsymbol{S}$ by $(s / 2, s / 2)$. For any edge $\boldsymbol{a}$ of $\boldsymbol{S}$, let $S_{1}(\boldsymbol{a})$ and $S_{2}(\boldsymbol{a})$ be the two adjacent squares to $\boldsymbol{a}$. See Figure 1 for a pictorial description. Let $\left\{a_{i}\right\}_{i=1}^{4}$ denote the four vertices of the rectangle $S_{1}(\boldsymbol{a}) \cup S_{2}(\boldsymbol{a})$. Let $Y(\boldsymbol{a})$ be the smallest square containing $\cup_{i=1}^{4} B\left(a_{i}, t\right)$, where $t$ is such that $\ell(t)<\ell(\sqrt{5} s) / 2$.

Definition 2.1. Any edge $\boldsymbol{a}$ of $\boldsymbol{S}$ is defined to be open if

1. there is at least one node of $\Phi$ in both the adjacent squares $S_{1}(\boldsymbol{a})$ and $S_{2}(\boldsymbol{a})$;

2. there are no eavesdropper nodes in $Y(\boldsymbol{a})$; and 


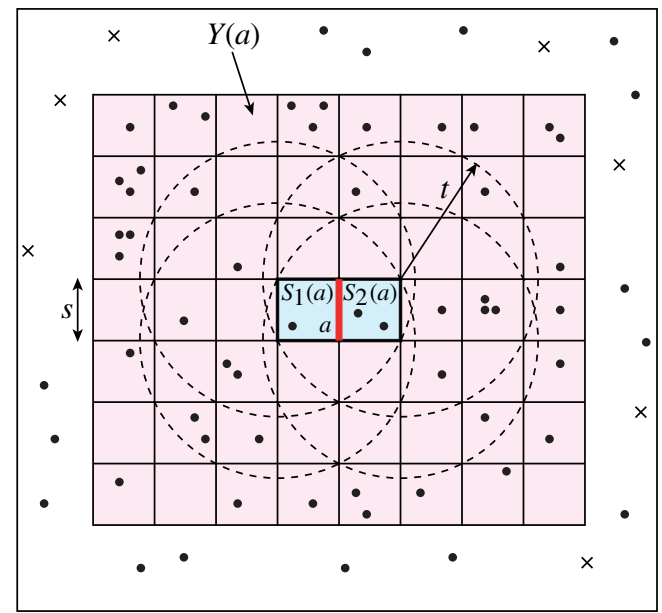

FIGURE 1: Open edge definition on a square lattice for supercritical regime.

3. for any legitimate node $x_{i} \in \Phi \cap\left(S_{1}(\boldsymbol{a}) \cup S_{2}(\boldsymbol{a})\right)$, the interference received at any legitimate node $x_{j} \in \Phi \cap\left(S_{1}(\boldsymbol{a}) \cup S_{2}(\boldsymbol{a})\right), I_{j}^{i}:=\sum_{k \in \Phi, k \neq i} \ell\left(x_{k}, x_{j}\right) \leq 1 / \gamma$.

An open edge is pictorially described in Figure 1 by edge $a$, where the black dots represent a legitimate node while a cross represents an eavesdropper node.

The next lemma allows us to tie up the continuum percolation on $\mathrm{SSG}^{e}$ to the bond percolation on the square lattice, where we show that if an edge $\boldsymbol{a}$ is open then all legitimate nodes lying in $S_{1}(\boldsymbol{a}) \cup S_{2}(\boldsymbol{a})$ can connect to each other.

Lemma 2.1. If an edge $\boldsymbol{a}$ of $\boldsymbol{S}$ is open then any node $x_{i} \in \Phi \cap\left(S_{1}(\boldsymbol{a}) \cup S_{2}(\boldsymbol{a})\right)$ can connect to any node $x_{j} \in \Phi \cap\left(S_{1}(\boldsymbol{a}) \cup S_{2}(\boldsymbol{a})\right)$ in $\mathrm{SSG}^{e}$.

Proof. For any $x_{i}, x_{j} \in \Phi \cap\left(S_{1}(\boldsymbol{a}) \cup S_{2}(\boldsymbol{a})\right), \mathrm{SINR}_{i j} \geq \ell(\sqrt{5} s) / 2$, since

$$
I_{j}^{i}:=\sum_{k \in \Phi, k \neq i} \ell\left(x_{k}, x_{j}\right) \leq \frac{1}{\gamma}
$$

for each $x_{i}, x_{j} \in \Phi \cap\left(S_{1}(\boldsymbol{a}) \cup S_{2}(\boldsymbol{a})\right)$. Moreover, since there are no eavesdropper nodes in $Y(\boldsymbol{a})$, the minimum distance between any eavesdropper node from any legitimate node in $\Phi \cap\left(S_{1}(\boldsymbol{a}) \cup S_{2}(\boldsymbol{a})\right)$ is at least $t$. Since $t$ is such that $\ell(t)<\ell(\sqrt{5} s) / 2$, clearly, $x_{i}, x_{j} \in$ $\Phi \cap\left(S_{1}(\boldsymbol{a}) \cup S_{2}(\boldsymbol{a})\right)$ are connected in $\mathrm{SSG}^{e}$. This completes the proof.

Definition 2.2. An open component of $S$ is the sequence of connected open edges of $S$.

Definition 2.3. A circuit in $\boldsymbol{S}$ or $\boldsymbol{S}^{\prime}$ is a connected path of $\boldsymbol{S}$ or $\boldsymbol{S}^{\prime}$ which starts and ends at the same point. A circuit in $S$ or $S^{\prime}$ is defined to be open/closed if all the edges on the circuit are open/closed in $S$ or $S^{\prime}$.

Some important properties of $\boldsymbol{S}$ and $\boldsymbol{S}^{\prime}$ which are immediate are as follows.

Lemma 2.2. If the cardinality of the open component of $\boldsymbol{S}$ containing the origin is infinite, then $\left|C_{x_{1}}\right|=\infty$.

Proof. The proof follows from Lemma 2.1. 
Lemma 2.3. ([3].) The open component of $\boldsymbol{S}$ containing the origin is finite if and only if there is a closed circuit in $\boldsymbol{S}^{\prime}$ surrounding the origin.

Hence, if we can show that the probability that there exists a closed circuit in $S^{\prime}$ surrounding the origin is less than 1, then it follows that an unbounded connected component exists in $\boldsymbol{S}$ with nonzero probability. Moreover, having an unbounded connected component in the square lattice $S$ implies that there is an unbounded connected component in SSG from Lemma 2.1. Next, we find a bound on $\lambda$ as a function of $\lambda_{E}$ such that the probability of having a closed circuit in $S^{\prime}$ surrounding the origin is less than 1. This is a standard approach used for establishing the existence of percolation in discrete graphs.

For an edge $\boldsymbol{a}$, let $A(\boldsymbol{a})=1$ if $\Phi \cap S_{i}(\boldsymbol{a}) \neq \varnothing, i=1,2$, and zero otherwise. Similarly, let $B(\boldsymbol{a})=1(=0)$ if $I_{j}^{i}:=\sum_{k \in \Phi, k \neq i} \ell\left(x_{k}, x_{j}\right) \leq 1 / \gamma$ for $x_{i}, x_{j} \in \Phi \cap\left(S_{1}(\boldsymbol{a}) \cup S_{2}(\boldsymbol{a})\right)$ (otherwise), and $C(\boldsymbol{a})=1(=0)$ if there are no eavesdropper nodes in $Y(\boldsymbol{a})$ (otherwise). Then by definition, the edge $\boldsymbol{a}$ is open if $D(\boldsymbol{a})=A(\boldsymbol{a}) B(\boldsymbol{a}) C(\boldsymbol{a})=1$.

Now we want to bound the probability of having a closed circuit surrounding the origin in $S$. Towards that end, we will first bound the probability of a closed circuit of length $n$, i.e. $P\left(D\left(\boldsymbol{a}_{1}\right)=0, D\left(\boldsymbol{a}_{2}\right)=0, \ldots, D\left(\boldsymbol{a}_{n}\right)=0\right)$ for all $n \in \mathbb{N}$ considering $n$ distinct edges. Let $p_{A}:=P\left(A\left(\boldsymbol{a}_{i}\right)=0\right)$ for any $i$. Since $\Phi$ is a PPP with density $\lambda, p_{A}=1-\left(1-\exp \left(-\lambda s^{2}\right)\right)^{2}$. Then we have the following intermediate results to upper bound $P\left(D\left(\boldsymbol{a}_{1}\right)=0, D\left(\boldsymbol{a}_{2}\right)=\right.$ $\left.0, \ldots, D\left(\boldsymbol{a}_{n}\right)=0\right)$.

Lemma 2.4. It holds that $P\left(A\left(\boldsymbol{a}_{1}\right)=0, A\left(\boldsymbol{a}_{2}\right)=0, \ldots, A\left(\boldsymbol{a}_{n}\right)=0\right) \leq p_{1}^{n}$, where $p_{1}:=$ $p_{A}^{1 / 4}$.

Proof. The proof follows from the fact that in any sequence of $n$ edges of $\boldsymbol{S}$ there are at least $n / 4$ edges such that their adjacent rectangles $S_{1}\left(\boldsymbol{a}_{e}\right) \cup S_{2}\left(\boldsymbol{a}_{e}\right)$ do not overlap. Therefore, $P\left(A\left(\boldsymbol{a}_{1}\right)=0, A\left(\boldsymbol{a}_{2}\right)=0, \ldots, A\left(\boldsymbol{a}_{n}\right)=0\right) \leq P\left(\cap_{e \in O} A\left(\boldsymbol{a}_{e}\right)=0\right)$, where $O$ is the set of edges for which their adjacent rectangles $S_{1}\left(\boldsymbol{a}_{e}\right) \cup S_{2}\left(\boldsymbol{a}_{e}\right)$ have no overlap, and $|O|=n / 4$. Since $S_{1}\left(\boldsymbol{a}_{e}\right) \cup S_{2}\left(\boldsymbol{a}_{e}\right), e \in O$ have no overlap, and events $A\left(\boldsymbol{a}_{e}\right)=0$ are independent for $\boldsymbol{a}_{e} \in O$, the result follows. This completes the proof.

Lemma 2.5. ([2, Proposition 2].) For $\int_{0}^{\infty} x \ell(x) \mathrm{d} x<\infty, P\left(B\left(\boldsymbol{a}_{1}\right)=0, B\left(\boldsymbol{a}_{2}\right)=0, \ldots\right.$, $\left.B\left(\boldsymbol{a}_{n}\right)=0\right) \leq p_{2}^{n}$, where

$$
p_{2}:=\exp \left(\frac{2 \lambda}{K} \int \ell(x) \mathrm{d} x-\frac{1}{\gamma K}\right)
$$

and $K$ is a constant.

Lemma 2.6. It holds that $P\left(C\left(\boldsymbol{a}_{1}\right)=0, C\left(\boldsymbol{a}_{2}\right)=0, \ldots, C\left(\boldsymbol{a}_{n}\right)=0\right) \leq p_{3}^{n}$ for some $p_{3}$ independent of $n$.

Proof. By definition, events $C\left(\boldsymbol{a}_{i}\right)$ and $C\left(\boldsymbol{a}_{j}\right)$ are independent if $Y\left(\boldsymbol{a}_{i}\right) \cap Y\left(\boldsymbol{a}_{j}\right)=\varnothing$. Consider a circuit $\mathcal{P}_{n}$ in $S$ of length $n$, with a subset $\mathcal{P}_{n}^{s} \subset \mathcal{P}_{n}$, where $\mathcal{P}_{n}^{s}=\left\{\boldsymbol{a}_{i}\right\}_{i \in \mathcal{I}}$, where for any $n, m \in \mathcal{L}, Y\left(\boldsymbol{a}_{n}\right) \cap Y\left(\boldsymbol{a}_{m}\right)=\varnothing$. Since $Y(\boldsymbol{a})$ occupies at most $(L+\lceil 2 t / s\rceil) \times(L+1+\lceil 2 t / s\rceil)$ rectangles of lattice $S$, where $L=2\lceil\sqrt{5}\rceil$, it follows that $|\mathcal{I}| \geq n / \psi$, where $\psi=8(L+$ $\lceil 2 t / s\rceil)^{2}-1$. Thus, $P\left(C\left(\boldsymbol{a}_{1}\right)=0, C\left(\boldsymbol{a}_{2}\right)=0, \ldots, C\left(\boldsymbol{a}_{n}\right)=0\right) \leq p_{3}^{n}$, where $p_{3}=P\left(C\left(\boldsymbol{a}_{i}\right)=\right.$ $0)^{1 / \psi}$ and $P\left(C\left(\boldsymbol{a}_{i}\right)=0\right)=\mathrm{e}^{-\lambda_{E} v\left(Y\left(\boldsymbol{a}_{i}\right)\right)}$. This completes the proof.

Lemma 2.7. It holds that $P\left(D\left(\boldsymbol{a}_{1}\right)=0, D\left(\boldsymbol{a}_{2}\right)=0, \ldots, D\left(\boldsymbol{a}_{n}\right)=0\right) \leq\left(\sqrt{p_{1}}+p_{2}{ }^{1 / 4}+\right.$ $\left.p_{3}^{1 / 4}\right)^{n}$. 
Proof. The proof follows from [2, Proposition 3], where $D(\boldsymbol{a})=1$ if $A(\boldsymbol{a}) B(\boldsymbol{a}) C(\boldsymbol{a})=1$.

Let $q:=\left(\sqrt{p_{1}}+p_{2}{ }^{1 / 4}+p_{3}{ }^{1 / 4}\right)$. The next lemma characterizes an upper bound on $q$ for which the probability of having a closed circuit in $S$ surrounding the origin is less than 1 .

Lemma 2.8. If $q<(11-2 \sqrt{10}) / 27$ then the probability of having a closed circuit in $\boldsymbol{S}^{\prime}$ surrounding the origin is less than 1.

Proof. For any circuit of length $n$, there are four possible choices of edges for the starting step and thereafter three choices for every subsequent step, except for the last step which is fixed since the circuit has to terminate at the starting point. Moreover, for a circuit containing the origin, the maximum possible distinct intersections with the $x$-axis are $n$. Thus, the number of possible circuits of length $n$ around the origin is less than or equal to $4 n 3^{n-2}$. From Lemma 2.7, we know that the probability of a closed circuit of length $n$ is upper bounded by $q^{n}$. Thus,

$$
P(\text { closed circuit around origin }) \leq \sum_{n=1}^{\infty} 4 n 3^{n-2} q^{n}=\frac{4 q}{3(1-3 q)^{2}},
$$

which is less than 1 for $q<(11-2 \sqrt{10}) / 27$.

Proof of Theorem 1.1. Following Lemmas 2.3 and 2.8, it is sufficient to show that $q$ can be made arbitrarily small for an appropriate choice of parameters. For any eavesdropper density $\lambda_{E}$, $p_{3}$ can be made arbitrarily small by choosing small enough $s$ and $t$. Depending on the choice of $s, p_{1}$ can be made arbitrarily small for large enough legitimate node density $\lambda$, and finally depending on the choice of $\lambda$, choosing small enough $\gamma, p_{2}$ can be made arbitrarily small. This completes the proof.

\section{Proof of Theorem 1.2}

In this section we are interested in the subcritical regime of percolation, i.e. obtaining a lower bound on $\lambda_{c}$ as a function of $\lambda_{E}$ for which percolation does not happen. We consider the case of $\gamma=0$, where $x_{i}$ and $x_{j}$ are connected in the SSG if

$$
\ell\left(d_{i j}\right)<\left(\frac{\ell\left(x_{i}, \mathrm{e}\right)}{1+\sum_{x_{j} \in \Phi, j \neq i} \ell\left(x_{j}, \mathrm{e}\right)}\right) \quad \text { for all } \mathrm{e} \in \Phi_{E} .
$$

If we can show that $\lambda_{c}>\lambda_{0}$ for $\gamma=0$, then since the SSG with $\gamma>0$ is contained in the SSG with $\gamma=0$, we have that for all $\gamma>0, \lambda_{c}>\lambda_{0}$. So the lower bound $\lambda_{0}$ for $\lambda_{c}$ obtained with $\gamma=0$ serves as a universal lower bound on the critical density $\lambda_{c}$ required for percolation. The interference power received at any eavesdropper, with respect to signal from $x_{k}$ is $I_{e}^{k}:=\sum_{x_{j} \in \Phi, j \neq k} \ell\left(x_{j}, \mathrm{e}\right)$.

For the case of $\gamma=0$, we proceed as follows. We tile $\mathbb{R}^{2}$ into a square lattice $\boldsymbol{M}$ with side $M$. Let $\boldsymbol{M}^{\prime}=\boldsymbol{M}+(M / 2, M / 2)$ be the dual lattice of $\boldsymbol{M}$ obtained by translating each edge of $\boldsymbol{M}$ by $(M / 2, M / 2)$. For any edge $\boldsymbol{e}$ of $\boldsymbol{M}$, let $S_{1}(\boldsymbol{e})$ and $S_{2}(\boldsymbol{e})$ be the two adjacent squares to $\boldsymbol{e}$. See Figure 2 for a pictorial description. Let $T_{1}(\boldsymbol{e})$ and $T_{2}(\boldsymbol{e})$ be the smaller squares of side $m$ contained inside $S_{1}(\boldsymbol{e})$ and $S_{2}(\boldsymbol{e})$, respectively, as shown in Figure 2, with centers identical to that of $S_{1}$ and $S_{2}$.

Definition 3.1. For any edge $\boldsymbol{e}$ of $\boldsymbol{M}$, we define three indicator variables $\tilde{A}(\boldsymbol{e}), \tilde{B}(\boldsymbol{e})$, and $\tilde{C}(\boldsymbol{e})$ as follows. 


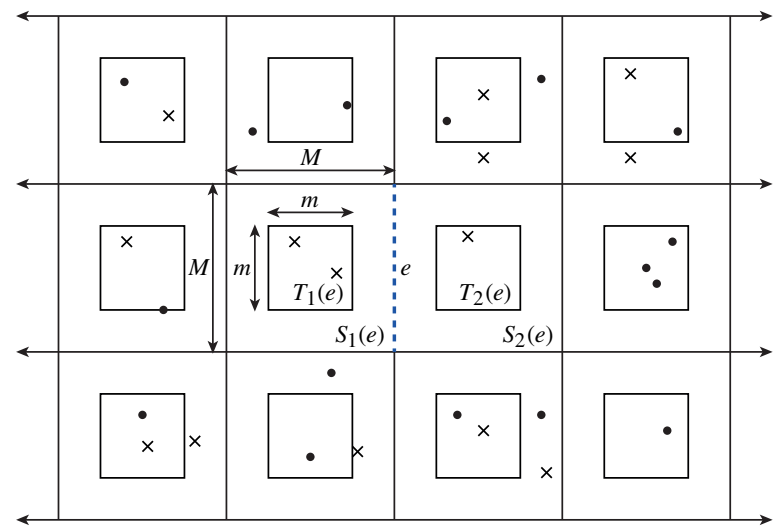

FIGURE 2: Open edge definition on a square lattice for subcritical regime.

1. $\tilde{A}(\boldsymbol{e})=1$ if there is at least one eavesdropper node of $\Phi_{E}$ in both the adjacent squares $T_{1}(\boldsymbol{e})$ and $T_{2}(\boldsymbol{e})$.

2. $\tilde{B}(\boldsymbol{e})=1$ if there are no legitimate nodes in $S_{1}(\boldsymbol{e})$ and $S_{2}(\boldsymbol{e})$.

3. $\tilde{C}(\boldsymbol{e})=1$ if for any eavesdropper node $e \in \Phi_{E} \cap\left(T_{1}(\boldsymbol{e}) \cup T_{2}(\boldsymbol{e})\right)$, the interference received from all the legitimate nodes $I_{e}:=\sum_{x_{k} \in \Phi} \ell\left(x_{k}, e\right) \leq c$.

Then an edge $\boldsymbol{e}$ is defined to be open if $\tilde{D}(\boldsymbol{e})=\tilde{A}(\boldsymbol{e}) \tilde{B}(\boldsymbol{e}) \tilde{C}(\boldsymbol{e})=1$. An open edge is pictorially described in Figure 2 by a dashed edge $\boldsymbol{e}$, where the black dots represent legitimate nodes and crosses represent eavesdropper nodes.

Lemma 3.1. For any $m$ and $c$, for large enough $M$, an edge $\left(x_{i}, x_{j}\right) \in \mathrm{SSG}$ cannot cross an open edge $\boldsymbol{e}$ of $\boldsymbol{M}$.

Proof. Let two legitimate nodes $x_{i}, x_{j} \in \Phi$ be such that the straight line between $x_{i}$ and $x_{j}$ intersects an open edge $\boldsymbol{e}$ of $\boldsymbol{M}$. Then by definition of an open edge, $x_{i}, x_{j} \notin\left(S_{1}(\boldsymbol{e}) \cup S_{2}(\boldsymbol{e})\right)$. Thus, the signal power between $x_{i}$ and $x_{j}$ is $\ell\left(d_{i j}\right)$, where $d_{i j}>M$. Moreover, the SINR between $x_{i}$ and any eavesdropper node $e \in\left(T_{1}(\boldsymbol{e}) \cup T_{2}(\boldsymbol{e})\right), \operatorname{SINR}_{i e} \geq \ell\left(d_{i e}\right) /(1+c)$, since edge $\boldsymbol{e}$ is open and hence $I_{e} \leq c$ for any $e \in\left(T_{1}(\boldsymbol{e}) \cup T_{2}(\boldsymbol{e})\right.$. Thus, choosing $M$ large enough, we can have $\ell\left(d_{i j}\right)<\operatorname{SINR}_{i e}$ for any $e \in\left(T_{1}(\boldsymbol{e}) \cup T_{2}(\boldsymbol{e})\right)$, and, hence, $x_{i}$ and $x_{j}$ cannot be connected directly in the SSG if the straight line between them happens to cross an open edge $\boldsymbol{e}$ of $\boldsymbol{M}$.

Definition 3.2. Consider a square box $B$. Then by $\{L \rightarrow R$ crossing of $B$ by $G\}$, we denote that there is a connected path of graph $G$ that crosses $B$ from left to right. Similarly, top-tobottom crossing is represented as $\{T \rightarrow D$ crossing of $B$ by $G\}$.

Lemma 3.2. If bond percolation happens on a square lattice $\boldsymbol{M}$ for large enough $M$ for which an edge $\left(x_{i}, x_{j}\right) \in \mathrm{SSG}$ cannot cross an open edge $\boldsymbol{e}$ of $\boldsymbol{M}$, then the connected component of the SSG is finite.

Proof. Consider a square box $B_{N}$ of side $N$ centered at the origin. Let $M$ be large enough such that an edge $\left(x_{i}, x_{j}\right) \in \mathrm{SSG}$ cannot cross an open edge $\boldsymbol{e}$ of $\boldsymbol{M}$. If bond percolation 
happens on a square lattice $\boldsymbol{M}$ then

$$
\lim _{N \rightarrow \infty} P\left(\text { there exists an } L \rightarrow R \text { and } T \rightarrow D \text { crossing of } B_{N} \text { by open edges of } \boldsymbol{M}\right)=1 .
$$

The proof of the theorem follows by contradiction. Let there be an infinite connected component in the SSG with probability 1 . Then, necessarily

$$
\lim _{N \rightarrow \infty} P\left(\text { there exists an } L \rightarrow R \text { and } T \rightarrow D \text { crossing of } B_{N} \text { by SSG }\right)=1 .
$$

Since $M$ is such that an edge $\left(x_{i}, x_{j}\right) \in \mathrm{SSG}$ cannot cross an open edge $\boldsymbol{e}$ of $\boldsymbol{M}$, (3.1) and (3.2) cannot hold simultaneously. This completes the proof.

Next, we show that for small enough density of legitimate nodes $\lambda$, bond percolation can happen on a square lattice $\boldsymbol{M}$ for large enough $M$ for which an edge $\left(x_{i}, x_{j}\right) \in$ SSG cannot cross an open edge $\boldsymbol{e}$ of $\boldsymbol{M}$.

Theorem 3.1. For large enough $M$ that ensures that $\left(x_{i}, x_{j}\right) \in \mathrm{SSG}$ cannot cross an open edge $\boldsymbol{e}$ of $\boldsymbol{M}$, bond percolation on $\boldsymbol{M}$ happens for a small enough density of legitimate nodes $\lambda$.

Proof. Similar to the proof in the supercritical regime, we need to show that the probability of having a closed circuit surrounding the origin in $\boldsymbol{M}$ is less than 1 . Towards that end, consider the probability of a closed circuit of length $n, P\left(\tilde{D}\left(\boldsymbol{e}_{1}\right)=0, \tilde{D}\left(\boldsymbol{e}_{2}\right)=0, \ldots, \tilde{D}\left(\boldsymbol{e}_{n}\right)=0\right)$, where $\tilde{D}\left(\boldsymbol{e}_{1}\right)=\tilde{A}\left(\boldsymbol{e}_{1}\right) \tilde{B}\left(\boldsymbol{e}_{1}\right) \tilde{C}\left(\boldsymbol{e}_{1}\right)$. Similar to Lemma $2.4, P\left(\tilde{A}\left(\boldsymbol{e}_{1}\right)=0, \tilde{A}\left(\boldsymbol{e}_{2}\right)=0, \ldots, \tilde{A}\left(\boldsymbol{e}_{n}\right)=\right.$ $0) \leq r_{1}^{n}$, where $r_{1}:=r_{A}^{1 / 4}$ and $\left.r_{A}=1-1-\exp \left(-\lambda_{E} m\right)\right)^{2}$ is the probability that there is no eavesdropper in either $T_{1}(\boldsymbol{e})$ or $T_{2}(\boldsymbol{e})$. Similarly, following Lemma $2.4, P\left(\tilde{B}\left(\boldsymbol{e}_{1}\right)=0\right.$, $\left.\tilde{B}\left(\boldsymbol{e}_{2}\right)=0, \ldots, \tilde{B}\left(\boldsymbol{e}_{n}\right)=0\right) \leq r_{2}^{n}$, where $r_{2}:=r_{B}^{1 / 4}$ and $r_{B}=1-\mathrm{e}^{-2 \lambda M^{2}}$ is the probability that there is at least one legitimate node of $\Phi$ in $S_{1}(\boldsymbol{e})$ or $S_{2}(\boldsymbol{e}), P\left(\tilde{C}\left(\boldsymbol{e}_{1}\right)=0, \tilde{C}\left(\boldsymbol{e}_{2}\right)=\right.$ $\left.0, \ldots, \tilde{C}\left(\boldsymbol{e}_{n}\right)=0\right) \leq r_{3}^{n}$, where $r_{3}=:=\exp \left(2 \lambda / K \int \ell(x) \mathrm{d} x-c / K\right)$ following Lemma 2.5 , and finally

$$
P\left(\tilde{D}\left(\boldsymbol{e}_{1}\right)=0, \tilde{D}\left(\boldsymbol{e}_{2}\right)=0, \ldots, \tilde{D}\left(\boldsymbol{e}_{n}\right)=0\right) \leq\left(\sqrt{r_{1}}+r_{2}^{1 / 4}+r_{3}^{1 / 4}\right)^{n}
$$

following Lemma 2.7. Let $r_{s}:=\sqrt{r_{1}}+r_{2}^{1 / 4}+r_{3}^{1 / 4}$.

Using Peierl's argument, bond percolation happens in $\boldsymbol{M}$ if $r_{s}<\epsilon$ for sufficiently small $\epsilon>0$. Let us fix such an $\epsilon>0$. Then, by choosing $m$ large enough, we can have $\sqrt{r_{1}}<$ $\epsilon / 3$. Moreover, for fixed $c$, let $M$ be large enough such that for any pair of legitimate nodes $x_{i}, x_{j} \notin\left(S_{1}(\boldsymbol{e}) \cup S_{2}(\boldsymbol{e})\right)$ for which the straight line between them intersects an open edge $\boldsymbol{e}$ of $\boldsymbol{M}, \ell\left(d_{i j}\right)<\mathrm{SINR}_{i e}$ for any $e \in\left(T_{1}(\boldsymbol{e}) \cup T_{2}(\boldsymbol{e})\right)$. Now, given $c, m$, and $M$, we can choose $\lambda$ small enough so that $r_{2}^{1 / 4}<\epsilon / 3$ and $r_{2}^{1 / 4}<\epsilon / 3$. Thus, we have $r_{s}<\epsilon$ as required for an appropriate choice of $m, M$ and $\lambda$.

Thus, from Theorem 3.1 and Lemma 3.2, we have for small enough legitimate node density $\lambda$, the SSG with $\gamma=0$ does not percolate.

\section{Acknowledgement}

S. Iyer's research was supported, in part, by the University grant commission career advancement scheme (UGC-CAS). 


\section{References}

[1] Dousse, O., Baccelli, F. And Thiran, P. (2005). Impact of interferences on connectivity in ad hoc networks. IEEE/ACM Trans. Networking 13, 425-436.

[2] Dousse, O. et al. (2006). Percolation in the signal to interference ratio graph. J. Appl. Prob. 43, 552-562.

[3] Grimmett, G. (1980). Percolation. Springer.

[4] Gupta, P. and Kumar, P. R. (2000). The capacity of wireless networks. IEEE Trans. Inf. Theory 46, $388-404$.

[5] HaengGi, M. (2008). The secrecy graph and some of its properties. In Proc. IEEE Internat. Symp. Inf. Theory ISIT 2008, IEEE, pp. 539-543.

[6] Meester, R. ANd Roy, R. (1996). Continuum Percolation. Cambridge University Press.

[7] Penrose, M. (2003). Random Geometric Graphs. Oxford University Press.

[8] Pinto, P. ANd Win, M. Z. (2012). Percolation and connectivity in the intrinsically secure communications graph. IEEE Trans. Inf. Theory 58, 1716-1730.

[9] Sarkar, A. and Haenggi, M. (2013). Percolation in the secrecy graph. Discrete Appl. Math. 161, 2120-2132.

[10] VAZE, R. (2012). Percolation and connectivity on the signal to interference ratio graph. In Proc. IEEE INFOCOM 2012, IEEE, pp. 513-521.

[11] Wyner, A. D. (1975). The wire-tap channel. Bell Syst. Tech. J. 54, 1355-1387. 